\title{
Superdiffusion in Si Crystal Lattice Irradiated by Soft X-Rays
}

\author{
A.J. JanaviČiuss ${ }^{a}$, S. Balakauskas ${ }^{b}$, V. Kazlauskiene $\dot{B}^{c}$, \\ A. Mekys ${ }^{d}$, R. Purlys ${ }^{d}$ AND J. Storasta ${ }^{c, d}$ \\ ${ }^{a}$ Faculty of Natural Sciences, Šiauliai University \\ P. Višinskio 19, 77156 Šiauliai, Lithuania \\ ${ }^{b}$ Semiconductor Physics Institute, A. Goštauto 11, 2600 Vilnius, Lithuania \\ ${ }^{c}$ Institute of Material Science and Applied Researches \\ Vilnius University, Saulètekio al. 9, bld. 3, 2040 Vilnius, Lithuania \\ ${ }^{d}$ Faculty of Physics, Vilnius University \\ Saulètekio al. 9, bld. 3, 2040 Vilnius, Lithuania \\ (Received June 25, 2007; revised version June 11, 2008)
}

We considered the reasons of superdiffusivity and measured profiles of boron and phosphorus in crystalline silicon at room temperature. The superdiffusivity or ultrafast diffusion of metastable vacancies at room temperature in $\mathrm{Si}$ crystal irradiated by soft X-rays was obtained experimentally. In this work, we presented experimentally obtained diffusion coefficients of singly and doubly negatively charged long-lived excited vacancies. These high concentration charged metastable vacancies (about $10^{13} \mathrm{~cm}^{-3}$ ) at room temperature can very fast diffuse changing electrical conductivity and the Hall mobility of carriers. We measured the superdiffusivity of negatively charged vacancies, generated by the Auger effect in the regions of the sample, which were not affected by X-rays. In this paper, we presented the obtained superdiffusion profiles of boron and phosphorus in crystalline silicon measured with secondary-ion mass spectrometer.

PACS numbers: 66.30.-h, 78.70.Ck

\section{Introduction}

We can assume that ultrafast diffusion or superdiffusion can be realized when the vacancies and impurity atoms are excited or out of equilibrium with the lattice [1]. Earlier very rapid thermal diffusion of indium in $\mathrm{HgCdTe}$ crystal activated by tungsten-halogen radiation was obtained experimentally [2]. The penetration of the fast component of diffusing indium atoms in this case weakly depended on time and temperature. This kind of superdiffusion occurred due to excited va- 
cancies generated by ultraviolet irradiation by $4.14 \mathrm{eV}$ photons. But only $10^{-4}$ or $10^{-3}$ part of indium atoms participated in this very fast diffusion or superdiffusion. The slow atomic diffusion, occurring through non-excited vacancies, depends on time and temperature in usual way. Several papers published in the last decade deal with superdiffusion generated in crystals by electrons $[3,4]$ with energies of $0.75 \div 7 \mathrm{MeV}$ or slow neutrons [5].

In our former paper we considered the problem of superdiffusivity theoretically [1]. We experimentally obtained long-lived (at room temperatures) and ultrafast diffusing excited vacancies [6] in crystalline silicon irradiated by soft X-rays. Excited vacancies can be produced by moving the atoms from the lattice sites into neighboring metastable states by passing through the energy barrier of $1 \mathrm{eV}$, or to interstitial states with energies $0.8 \mathrm{eV}$ [7], as a result of the Coulomb interaction after the ejection of the Auger electrons [7]. These atoms can penetrate into metastable states through barrier of height $1.5 \mathrm{eV}$ [7]. For displacement into interstitial state they must penetrate through an additional barrier $0.5 \mathrm{eV}[7]$. The superdiffusion of irradiated vacancies is a result of electrons transitions between conductivity band, valence band and vacancies, and it is also influenced by local lattice vibrations [8]. The local heating of atoms surrounding the excited vacancies can increase local temperature up to $1300 \mathrm{~K}$ [6]. It is very important that excited negative vacancies can stay stable in Si crystal (with dimensions $3.33 \times 3.06 \times 3.09 \mathrm{~mm}^{3}$ ) during $1.5 \mathrm{~h}$ after irradiation and take part in very fast diffusion. We obtained very interesting results from the Hall measurements.

The abrupt changes of the Hall mobility [9], after switching off X-rays irradiation and its restoring after a $25 \mathrm{~h}$ time interval, indicate some processes associated with metastable defects in silicon. The obtained singly and doubly charged excited vacancies [9] have diffusion coefficients $10^{4}$ times larger than those of vacancies obtained by thermal heating [7]. This result can be explained only by differences between thermally generated and irradiated vacancies, which are in excited metastable states.

A very long lifetime existence of excited negative vacancies [9] (about $9 \mathrm{~h}$ ) and their superdiffusion at room temperature $[6,9,10]$ creates the conditions for realizing of the superdiffusivity of impurities in excited Si crystals [11] and possibility of production of new microprocessors and solar cells. Mathematical modeling and investigation of excited vacancy diffusion in Si crystal can be useful for interpretation of experiments and projecting of new devices.

These metastable vacancies have a sufficiently high concentration (about $10^{13} \mathrm{~cm}^{-3}$ ) at room temperature and can drive in of impurities in the silicon crystal by superdiffusion. We measured superdiffusivity of boron and phosphorus through vacancies, generated by Auger's effect in the surface region of the sample, which was irradiated by soft X-rays. The measurements of boron and phosphorus profiles after superdiffusion in crystal silicon at room temperature were done using a secondary-ion mass spectrometer. 


\section{Experimental details}

We provided the generation of positive vacancies in the $p$-Si sample (Hall mobility $\left.\mu_{0 \mathrm{H}}=237 \pm 5 \mathrm{~cm}^{2} /(\mathrm{V} \mathrm{s}), p_{0}=4 \times 10^{13} \mathrm{~cm}^{-3}, \sigma_{0}=1.51 \times 10^{-3} \Omega^{-1} \mathrm{~cm}^{-1}\right)$ using the X-ray tube of a diffractometer DRON 2.0 (Russian device) with a copper anode. The intensity of the X-ray beam and vacancies generation in the Si sample decrease exponentially

$$
I_{\mathrm{B}}(E)=I_{0}(E) \mathrm{e}^{-\mu(E) x} .
$$

The absorption coefficient $\mu(E)$ was determined using the following formulae for the cross-section $\sigma_{f}[12]$ of photoelectric effect and the X-ray absorption coefficient $\mu$ :

$$
\sigma_{f}=\frac{C Z^{k}}{(\hbar \omega)^{n}}, \quad \mu=n_{0} \sigma_{f},
$$

where $C$ is a constant, $k=4, n=3.5$ for X-rays, and $n_{0}=5 \times 10^{22} \mathrm{~cm}^{-3}$ is the concentration of atoms in Si crystal. Using the cross-section for $\mathrm{Al}$ [12] we obtain $C=1.728 \times 10^{-81} \mathrm{~m}^{2} \mathrm{~J}^{3.5}$. Then for Si irradiated by $K_{\alpha}$ photons of $\mathrm{Cu}$ anode $(E=8.986 \mathrm{keV})$ we obtained the cross-section $(2) \sigma_{f}=8.81 \times 10^{-25} \mathrm{~m}^{2}$ for photoelectric emission of electrons and the absorption coefficient $\mu=439 \mathrm{~cm}^{-1}$.

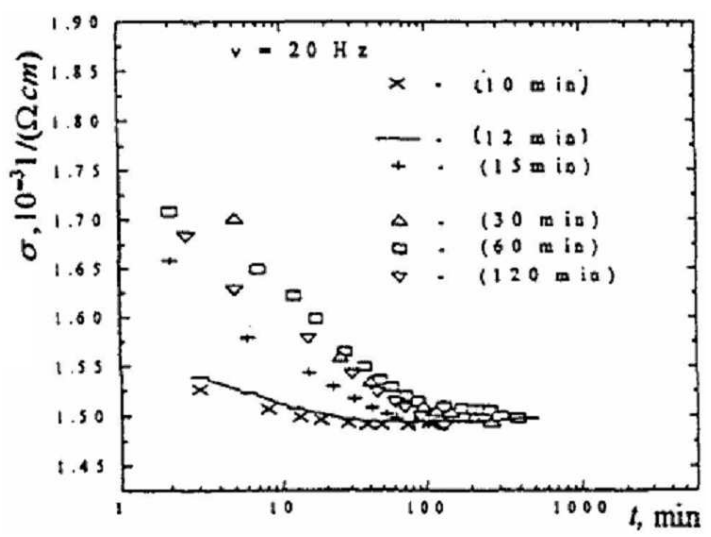

Fig. 1. The dependence of the electrical conductivity $\sigma$ of the sample upon the time $t$ after its saturation by vacancies.

The Compton cross-section is insignificant. Also we can get weak absorption $\mu=12.8 \mathrm{~cm}^{-1}$ for photons of X-rays with $22 \mathrm{keV}$ energies. The obtained results show that only the photons with maximum energies $(25 \mathrm{keV})$ can leave the sample of thickness $d=3.09 \mathrm{~mm}$. The majority of photons have energies less than $10 \mathrm{keV}$ and most photons are absorbed in the surface region. X-rays cannot generate vacancies directly by moving the interstitial atoms because the dislocation energy $15.8 \mathrm{eV}[13]$ of Si atoms is sufficiently high.

The conductivity of the sample was measured by the Hewlett-Packard (4284 A $20 \mathrm{~Hz}-1 \mathrm{MHz}$ Precision LCR meter) bridge. We presented the curves of the 
time dependence of the measured conductivity after different periods of irradiation. These curves have been used to determine the time of saturation of the sample with vacancies (curves are presented in Fig. 1 for different periods of irradiation). Also, these curves indicate that the sample is saturated by vacancies of one type. By changing the irradiation periods, we found the time of saturation of the sample with negatively charged vacancies of high mobility [9]: $t_{\mathrm{s}}=15 \mathrm{~min}$. We see that the saturation time cannot be measured with high accuracy, and our calculations are only approximate.

\section{Results}

At first we must calculate the number of the vacancies that were generated at the surface of the sample. The conductivity of the sample of Si doped by boron can be expressed as

$$
\sigma=p e \mu_{p}
$$

where $p$ is the number of holes per cubic centimeter and $\mu_{p}$ is their mobility. At a given temperature and mobility, the conductivity depends on the hole concentration. Using $\sigma_{1}$ and $p_{1}$ values given in [14] $\left(\sigma_{1}=0.01 \Omega^{-1} \mathrm{~cm}^{-1}\right.$ when the concentration of boron acceptors $p_{1}=3.1 \times 10^{14} \mathrm{~cm}^{-3}$ at $\left.T=300 \mathrm{~K}\right)$, and the measured conductivity $\sigma_{2}=1.5 \times 10^{-3} \Omega^{-1} \mathrm{~cm}^{-1}$ after migration of irradiated vacancies from the volume to the surface of the crystal (see Fig. 1), from (3) we obtained the following initial concentration of holes: $p_{2}=4.65 \times 10^{13} \mathrm{~cm}^{-3}$.

After the irradiation, conductivity (3) of the sample increases up to $1.71 \times 10^{-3} \Omega^{-1} \mathrm{~cm}^{-1}$ and adding the concentration of holes $\Delta p_{2}$ produced by irradiation to $p_{2}$ density of hole becomes $p_{2}+\Delta p_{2}=5.33 \times 10^{13} \mathrm{~cm}^{-3}$. We can use the additional density of holes $\Delta p_{2}=0.682 \times 10^{13} \mathrm{~cm}^{-3}$ for evaluation of the number of vacancies introduced into the volume $V$ of the sample during irradiation

$$
N_{V}=\Delta p_{2} V, \quad N_{V}=2.13 \times 10^{11} .
$$

In this experiment the anode voltage $22 \mathrm{kV}$ of the $\mathrm{X}$-ray tube and anode current $15 \mathrm{~mA}$ was used. The energy flux of the incident radiation measured by the scintillation counter was $\Phi=1.22 \times 10^{15} \mathrm{eV} \mathrm{cm}^{-2} \mathrm{~s}^{-1}$. Vacancies can be created by photons absorbed in the surface layer. The surface density of the atoms on the surface of $\mathrm{Si}[14]$ is $N=6.8 \times 10^{10} \mathrm{~cm}^{-2}$. Using the lattice constant of Si $a=0.543 \times 10^{-8} \mathrm{~cm}[14]$ and the absorption coefficient calculated from (2) $\left(\mu=439 \mathrm{~cm}^{-1}\right)$, we can find the fraction of photons absorbed in the surface layer

$$
P=1-\exp (-\mu a) \text {. }
$$

Only this fraction of photons can initiate the generation of vacancies. Now, we can approximately evaluate the energy used for produced vacancies

$$
\Delta E=\Phi P S t_{1}, \quad \Delta E=2.24 \times 10^{11} \mathrm{eV},
$$

where $S=0.333 \times 0.306 \mathrm{~cm}^{2}$ is the area of the irradiated side of the crystal, $t_{1}=900 \mathrm{~s}$ is the minimal time of irradiation necessary for the saturation of the 
sample with vacancies. Now from (6) and (4) we can find the formation energy of one negatively charged vacancy. $\Delta E / N_{V}=1.05 \mathrm{eV}$ at the surface of sample. The number of vacancies introduced into the sample can be expressed using a slightly modified formula for the number of impurity atoms introduced by the diffusion process [15]:

$$
N_{V}=0.549 N_{\mathrm{s} 1} x_{01}, \quad x_{01}=1.616 \sqrt{D_{\mathrm{v}} t_{0}}, \quad N_{\mathrm{s} 1}=0.5 N_{V \mathrm{~s}}=2.13 \times 10^{11} .(7)
$$

$N_{\mathrm{s} 1}$ is the concentration of impurity atoms at the surface in a one-dimensional model of impurities diffusion from the constant source [16], $x_{01}=$ $0.309 \mathrm{~cm}$ is its maximum penetration depth or the length of the sample edge in the case of vacancy diffusion. From this, we can obtain the concentration of vacancies at the surface of the irradiated side $N_{V \mathrm{~s}}=2.51 \times 10^{12} \mathrm{~cm}^{-1}$ taking into account that only half of them will move changing charges into the bulk of the sample. In formula (1), $D_{\mathrm{v}}$ is the vacancy diffusion coefficient, $t_{0}$ is the time of saturation of the sample with the vacancies. In Fig. 1 we can see that the irradiation time needed for saturation of the sample with negative vacancies is equal to $t_{1}=15 \mathrm{~min}$. During this minimum irradiation time the maximum number of the negatively charged vacancies is introduced into the sample. Now, from Eq. (1) we can obtain diffusion coefficient of vacancies in Si at room temperature (about $290 \mathrm{~K})$

$$
D_{\mathrm{v}}=5.30 \times 10^{-5} \mathrm{~cm}^{2} \mathrm{~s}^{-1} \text {. }
$$

From Fig. 1 we can see that in all experiments with periods of irradiation longer than 15 min the saturation of the sample with vacancies was achieved and that the vacancies acting on the conductivity left the sample during the same time $t=87 \mathrm{~min}$. This process can be described by drive in or second stage diffusion of vacancies from the samples surface [15]. For the second-stage diffusion [15], we have the following relation between the constant vacancy concentration $N_{\mathrm{s} 1}$ on the irradiated surface during time $t_{1}$ of the first-stage diffusion (until saturation of the sample with the vacancies) and vacancies concentration $N_{\mathrm{s} 2}$ at the surface after time $t$ of the second stage of diffusion until vacancies leave the sample

$$
\frac{N_{\mathrm{s} 2}}{N_{V \mathrm{~s}}}=\frac{\left(D_{\mathrm{v}} t_{1}\right)^{1 / 3}}{\left(D_{\mathrm{v}} t_{1}+D_{2} t\right)^{1 / 3}} .
$$

Taking into account

$$
\frac{N_{\mathrm{s} 2}}{N_{V \mathrm{~s}}}=\frac{0.5 p_{2}}{p_{2}+\Delta p_{2}}
$$

from (9) and (10) we obtain the time $t=166 \mathrm{~min}$ necessary for escape of vacancies from the sample. Comparing this result with Fig. 1 we can see that this result approximately coincides with the experiment.

Using the known concentration of Si atoms $5 \times 10^{22} \mathrm{~cm}^{-3}[13]$ and (6), we can find the relative concentration of singly charged vacancies $N_{\mathrm{r}}=1.35 \times 10^{-10}$. Then using the known equation [10]: 


$$
D=D_{\mathrm{v}} N_{\mathrm{r}},
$$

we find the self-diffusion coefficient $7.15 \times 10^{-15} \mathrm{~cm}^{2} \mathrm{~s}^{-1}$ in crystalline Si at room temperature.

The obtained result (8) $D_{\mathrm{v}}$ for vacancies diffusion can be compared with the diffusion coefficients $D_{1 \mathrm{v}}=1.48 \times 10^{-14} \mathrm{~cm}^{2} \mathrm{~s}^{-1}$ and $D=7.28 \times 10^{-4} \mathrm{~cm}^{2} \mathrm{~s}^{-1}$ reported in [13] for temperatures $T_{1}=160 \mathrm{~K}$ and $T_{2}=1685 \mathrm{~K}$. Our result $D_{\mathrm{v}}=5.30 \times 10^{-5} \mathrm{~cm}^{-5} \mathrm{~s}^{-1}$ obtained for the charged vacancies is close to $D_{2 \mathrm{v}}$. Diffusion of vacancies and interstitials is important for electrical conductivity of semiconductors [17]. The obtained results show the possibility of introducing boron and phosphorus into silicon using X-rays irradiation at room temperature. This allows producing high quality microelectronic devices using the diffusion of impurities through vacancies irradiated by X-rays. Also in this paper we presented new method of determination of the diffusion coefficients for charged vacancies at room temperature. Values of the enthalpy of migration $\Delta E_{\mathrm{m}}$ are the same as the energy required to break the bonds between the atoms at the surface of Si. This energy for $\mathrm{Si}$ is from 1 to $1.5 \mathrm{eV}$ [7] and in the case of singly charged vacancies it is approximately equal to the vacancy formation energy $0.946 \mathrm{eV}$. Using the presented method for different temperatures, the enthalpy of vacancy migration and the pre-exponential factor can be obtained with sufficient accuracy. The presented method has practical importance, since vacancy migration is usually the dominant mechanism of atomic diffusion and crystal growth.

\section{Superdiffusivity of boron and phosphorus in Si crystal}

We applied the superdiffusion of charged vacancies irradiated by soft $\mathrm{X}$-rays for drive in of formerly introduced impurities by thermal diffusion. From the Hall conductivity investigations and successful drive in experiments with $\mathrm{Pt}$ introduction into silicon, using soft X-rays irradiation, we defined possibility of impurities introduction by irradiation till $400^{\circ} \mathrm{C}$ temperatures.

We realized thermal introduction at first stage of diffusion of phosphorus and boron into $p$-type silicon (specific resistance $2000 \Omega \mathrm{cm}$ ) at $1150^{\circ} \mathrm{C}$ with duration $10 \mathrm{~min}$. We realized second stage of diffusion by $5 \mathrm{~h}$ irradiation of sample using DRON-2 (Russian device) with soft X-rays (with $\mathrm{Cr}$ anode voltage $9 \mathrm{kV}$ and current $23 \mathrm{~mA}$ ).

We performed secondary-ion mass spectroscopy (SIMS) (the Cameca Riber System MIQ 156) to obtain the concentration profiles of $\mathrm{P}$ and B atoms in Si wafers as functions of depth from the Si surface. The results are shown in Figs. 2 and 3. SIMS measurements were performed using the primary ion $\left(\mathrm{Ar}^{+}\right)$beam with an energy of $5 \mathrm{keV}$. Calibrated standards are very important for accurate SIMS measurement. The time-to-depth conversion was made after the sputter crater depth analysis. Another instrumentation effect that complicates SIMS analysis is the edge or wall effect. To obtain good depth resolution it is important that only the signal from the flat, bottom portion of the sputtered crater was analyzed. Atoms 
are also ejected from the crater bottom as well as from the side walls during sputtering. Using electronic gating of the secondary ion yield signal or a lens system, it is possible to detect only those ions from the central part of the crater. The accuracy of the depth scale may only be around $15 \%$.

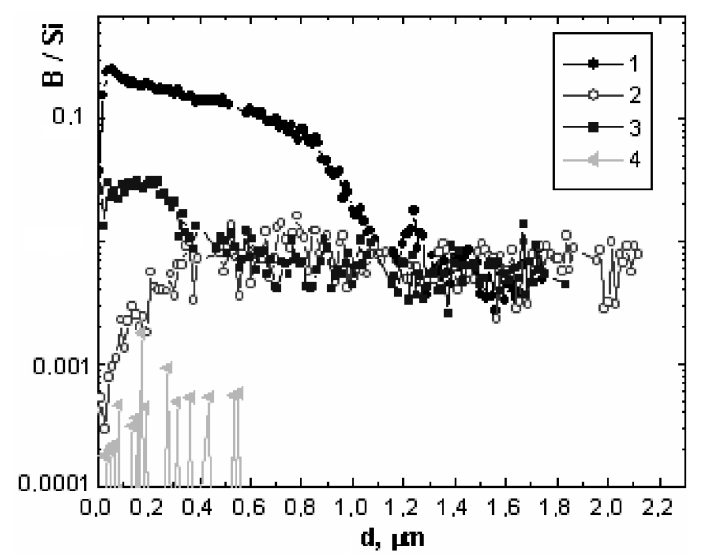

Fig. 2. B and Si ratios measured by SIMS is presented graphically: 1 - profile after thermodiffusion, 2 - profile after $5 \mathrm{~h}$ irradiation ( $\mathrm{Cr}$ anode, voltage $9 \mathrm{kV}$, current $23 \mathrm{~mA}$ ), 3 - profile after polishing $0.4 \mu \mathrm{m}, 4$ - profile in control sample.

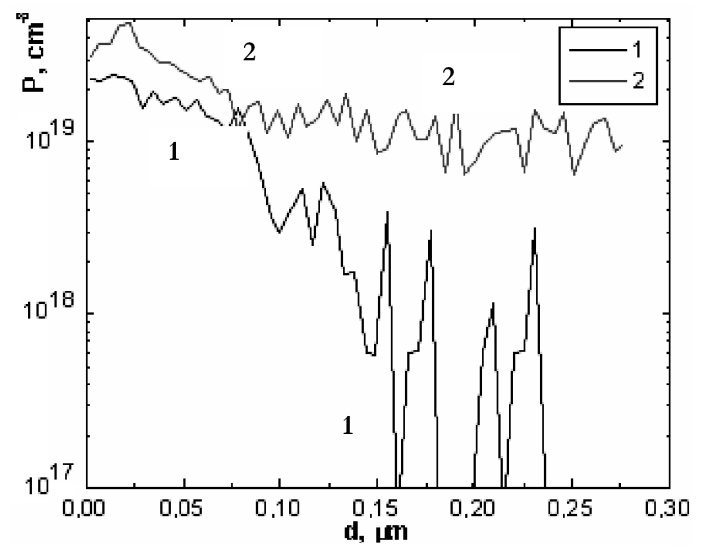

Fig. 3. P concentrations measured by SIMS is presented graphically: 1 - profile after thermodiffusion, 2 - profile after $5 \mathrm{~h}$ irradiation.

The Auger process in $p$-type silicon in this case [18] can generate only the positive charged vacancies $\mathrm{V}^{+}, \mathrm{V}^{++}$with energy levels in the silicon band gap close to the valence band [19]. In the result of electrons transitions from valence band into positive vacancies, their charges become negative and the vacancies levels are picked up. In this case positive vacancies are transforming to negative 
and reconstructing broken bonds getting energy from lattice [19] and transitions of electrons. Those excited negative vacancies, taking part in superdiffusion processes, were found in conductivity measurements of Si crystal irradiated by soft $\mathrm{X}$-rays $[6,9]$. Before and after lattice relaxation the levels [19] of $\mathrm{V}^{--}$and $\mathrm{V}^{-}$ are significantly upper $(0.6 \mathrm{eV}, 0.3 \mathrm{eV}$ consequently $)$ than two levels $\mathrm{V}^{+}, \mathrm{V}^{++}$ which are close to valence band. In this case positive vacancies are increasing the $p$ conductivity. Fast diffusing negative vacancies are generated by electrons transitions from valence band into positive charged vacancies $\mathrm{V}^{++}, \mathrm{V}^{+}$. Diffusing from the surface fast vacancies $\mathrm{V}^{--}, \mathrm{V}^{-}$are pushing the negative ions of substitution atoms of boron deeper into silicon sample. The thermal diffusion profiles of boron and phosphorus in silicon and profiles after irradiation of sample with $\mathrm{X}$-rays are presented in Figs. 2 and 3. The obtained profile for boron essentially differs from profile obtained by thermodiffusion, where we have maximum concentration of boron at the surface of sample. The diffusion of phosphorus in silicon is associated with singly and doubly-charged acceptor vacancies $\mathrm{V}^{2-}$. The coupled impurity-vacancy pair $\left(\mathrm{P}^{+} \mathrm{V}^{2-}\right)$ dissociates to electron and $\mathrm{P}^{+}, \mathrm{V}^{-}$. The negative vacancies significantly enhance diffusion of phosphorus in silicon and realize diffusion at room temperature. The diffusion profile for phosphorus introduced by irradiation in silicon is presented in Fig. 3. Neutral vacancies also can take part in diffusion of boron and phosphorus.

\section{Model calculations and discussion}

We prepared $p$-Si boron doped sample (the Hall mobility $\mu_{0 \mathrm{H}}=237 \pm$ $\left.5 \mathrm{~cm}^{2} /(\mathrm{V} \mathrm{s}), p_{0}=4 \times 10^{13} \mathrm{~cm}^{-3}, \sigma_{0}=1.51 \times 10^{-3} \Omega^{-1} \mathrm{~cm}^{-1}\right)$ with planar contacts. We obtained from the time dependence of the experimentally measured conductivity $1 / R$ in Fig. 4, the times $t_{\mathrm{s}}$ of saturation of the sample with singly and doubly negatively charged vacancies (irradiation conditions were realized using copper anode voltage $11 \mathrm{kV}$ and current $50 \mathrm{~mA}$ ). We deduced [6, 9] that the sample is saturated with the fast negative vacancies of two types. Considering the conductivity dependence on $\sqrt{t_{\mathrm{s}}}$, presented in Fig. 4 , where $t_{\mathrm{s}}$ is the saturation time of the sample by vacancies (the value of the saturated conductivity must be proportional to the penetration depth (1) of charged vacancies and $\sqrt{t_{\mathrm{s}}}$ ). We see that the saturation time $t_{\mathrm{s} 1}=200$ min may be obtained for the sample with singly charged vacancies of high mobility [9] and saturation time $t_{\mathrm{s} 2}=415$ min for slower doubly negatively charged vacancies [9]. It was only our assumption made using the same publications presented in $[9,10]$. We obtained that diffusing vacancies had negative charge at room temperature from the Hall mobility measurements [9].

At first we must calculate the number of vacancies generated on the surface of the sample. The bulk conductivity of the sample doped by boron may be expressed as $\sigma_{1}=p_{1} e \mu_{p}$ where $p_{1}$ is the number of holes per cubic centimeter and $\mu_{p}$ is their mobility. For the same temperature and different concentration of holes $p_{2}$ we have $\sigma_{2}=p_{2} e \mu_{p}$. Then using the result [9] of conductivity measurements presented in 


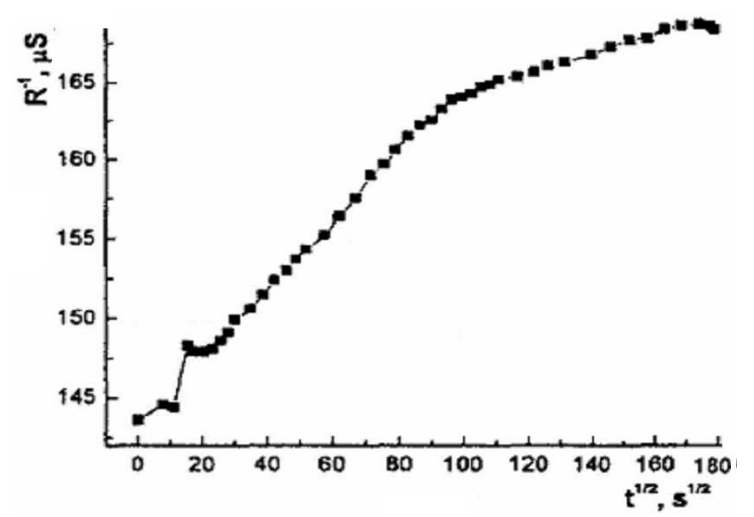

Fig. 4. The dependence of the conductivity $R^{-1}$ on the square root of the irradiation time $t$.

Fig. 4 before the irradiation of sample $\sigma_{1}=0.01 \Omega^{-1} \mathrm{~cm}^{-1}$ and the concentration of boron (acceptors) [14] $p_{1}=3.1 \times 10^{14} \mathrm{~cm}^{-1}$ for this case at $T=300 \mathrm{~K}$ and Eq. (3) we found the initial concentration of holes $p_{2}=4.49 \times 10^{13} \mathrm{~cm}^{-3}$.

After irradiation time $t_{\mathrm{s} 1}=200 \mathrm{~min}$, the sample of $\mathrm{Si}$ was saturated by diffusing singly charged vacancies and not saturated by doubly charged vacancies. Then the conductivity of the sample increases up to $1 / R=163 \mu \mathrm{S}$ and assuming that the conductivity increase is proportional to additional concentration of holes, produced by singly charged vacancies $\Delta p_{-}^{\mathrm{s}}$ (after irradiation time $t_{\mathrm{s} 1}=200 \mathrm{~min}$ ) and doubly negatively charged vacancies $\Delta p_{--}$, we obtain $p_{2}+\Delta p_{-}^{\mathrm{s}}+\Delta p_{--}=$ $5.15 \times 10^{13} \mathrm{~cm}^{-3}$. The increase in density of holes $\Delta p_{-}^{\mathrm{s}}+\Delta p_{--}=0.66 \times 10^{13} \mathrm{~cm}^{-3}$ can be used for evaluation of the amount of introduced fastest singly and doubly charged vacancies in the volume $V=0.309 \times 0.333 \times 0.306 \mathrm{~cm}^{3}$ of the sample. After the full saturation $\left(t_{\mathrm{s} 2}=415 \mathrm{~min}\right)$ from the following relation:

$$
\frac{\Delta \sigma_{2}}{\Delta \sigma^{\mathrm{s}}}=\frac{\Delta p_{-}^{\mathrm{s}}+\Delta p_{--}}{\Delta p_{-}^{\mathrm{s}}+\Delta p_{--}^{\mathrm{s}}},
$$

where $\Delta \sigma^{\mathrm{s}}=0.25 \times 10^{-3} \Omega^{-1} \mathrm{~cm}^{-1}$ is the increase in conductivity after full saturation of Si sample by negative vacancies and $\Delta \sigma^{\mathrm{s}}=0.2 \times 10^{-3} \Omega^{-1} \mathrm{~cm}^{-1}$ is the conductivity increase after saturation by singly negatively charged vacancies. Then from the last expressions we have

$$
\begin{aligned}
& \Delta p_{-}^{\mathrm{s}}+\Delta p_{--}^{\mathrm{s}}=0.825 \times 10^{13} \mathrm{~cm}^{-3}, \\
& \Delta p_{-}^{\mathrm{s}}+\Delta p_{--}=0.66 \times 10^{13} \mathrm{~cm}^{-3} .
\end{aligned}
$$

Taking into account (1), we find

$$
\frac{\Delta p_{--}}{\Delta p_{--}^{\mathrm{s}}}=\left(\frac{200}{415}\right)^{1 / 2}
$$

After solving of the system (13), (14) the concentrations of singly and doubly charged vacancies after saturation of the sample were obtained 
$\Delta p_{-}^{\mathrm{s}}=0.286 \times 10^{13} \mathrm{~cm}^{-3}, \quad \Delta p_{--}^{\mathrm{s}}=0.539 \times 10^{13} \mathrm{~cm}^{-3}$.

We irradiated the $\mathrm{Si}$ sample of thickness $0.309 \mathrm{~cm}$ from the side $0.333 \times 0.306 \mathrm{~cm}^{2}$. Then we evaluated the number of produced singly charged vacancies $\Delta N_{-}$and the number of singly and doubly charged vacancies

$$
\begin{aligned}
& \Delta N_{-}=\Delta p_{-}^{\mathrm{s}} V, \quad \Delta N_{-}=0.9 \times 10^{11} \mathrm{~cm}^{-3}, \\
& \Delta N_{--}=\frac{1}{2} \Delta p_{--}^{\mathrm{s}} V, \quad \Delta N_{--}=1.7 \times 10^{11} \mathrm{~cm}^{-3} .
\end{aligned}
$$

The number of vacancies introduced into the sample can be expressed using the nonlinear diffusion theory [15] $\Delta N_{\mathrm{v}}=0.5492 N_{\mathrm{s} 1} x_{01}, N_{\mathrm{s} 1}=0.5 N_{\mathrm{vs}}$. Here $N$ is the concentration of vacancies at the surface of the irradiated side $\left(N_{\mathrm{S} 1}\right.$ is the concentration of impurity atoms on the surface after diffusion from the constant source [15] in one-dimensional approach), $x_{01}=0.309 \mathrm{~cm}$ is the length of the sample edge or penetration depth of the vacancies (1). In the latter equation, $D_{\mathrm{v}}$ is the diffusion coefficient of vacancies and $t_{1}$ is the time of saturation of the sample with vacancies. From Fig. 4 and the results of the measurements, we can see that the irradiation time for saturation of the sample with singly charged vacancies reaches $t_{1}=200 \mathrm{~min}$ and with doubly charged vacancies $t_{2}=415 \mathrm{~min}$. During the time $t_{1}=200 \mathrm{~min}$, the maximum number of singly charged vacancies in the sample is introduced. Now, from (1) we can get the diffusion coefficients of singly and doubly charged vacancies in $\mathrm{Si}$ at room temperature (about $290 \mathrm{~K}$ ) as

$$
D_{-}=3.05 \times 10^{-6} \mathrm{~cm}^{2} / \mathrm{s}, \quad D_{--}=1.47 \times 10^{-6} \mathrm{~cm}^{2} / \mathrm{s} \text {. }
$$

We can compare the obtained results with expression of the diffusion coefficient of vacancies in Si obtained from low- and high-temperature experiments [7]:

$$
D_{\mathrm{v}}=3 \exp (-(1.0 \mathrm{eV}) / k T)+10^{-6} \exp (-(0.2 \mathrm{eV}) / k T) \mathrm{cm}^{2} / \mathrm{s} \text {. }
$$

From this formula for the room temperature $T=300 \mathrm{~K}$ we obtained

$$
D_{\mathrm{v}}=4.4 \times 10^{-10} \mathrm{~cm}^{2} / \mathrm{s} .
$$

Now we can make an important conclusion that diffusion conditions in the irradiated sample of Si are essentially different from thermal diffusion.

From (18) we can obtain the diffusion coefficients like in (16) and (17) only for temperature about $800 \mathrm{~K}$. We may conclude that the irradiated sample and irradiated vacancies are in some excited states and superdiffusivity through excited and very fast negative vacancies can be realized.

We arrived at the same conclusion for the Si sample after measurements of abrupt changes of conductivity and the Hall mobility after irradiation [20]. Here we had a complicated decrease in conductivity, which is caused by time dependence of the structure of metastable defects in silicon [9].

\section{Conclusions}

Using soft X-rays, we obtained long-lived ultrafast excited vacancies, which were used for introduction of boron and phosphorus into silicon by the second- 
-stage diffusion at room temperature. We realized the first stage of diffusion of boron and phosphorus in $p$-type silicon by thermodiffusion at $1150^{\circ} \mathrm{C}$ during 10 min. The presented in Figs. 2 and 3 very slowly lowering profiles, measured with SIMS after $5 \mathrm{~h}$ irradiation with soft X-rays, show that we realized the fast drive in diffusion or superdiffusion of boron and silicon in crystal silicon.

The soft X-rays photons are mostly absorbed in the surface region and have sufficient energy [10] for producing photoelectrons and Auger electrons in the inner $K, L, M$ shells of Si. They break chemical bonds at the surface of the Si sample and generate the excited vacancies. The interstitial atoms cannot be generated by X-rays directly, because the dislocation energy $15.8 \mathrm{eV}$ [13] of atoms in $\mathrm{Si}$ is sufficiently high. The Auger processes in $p$-type silicon in this case [18] can generate only the positive charged vacancies $\mathrm{V}^{+}, \mathrm{V}^{++}$with energy levels in the silicon band gap, close to the valence band [19]. As a result of electron transitions from valence band to positive vacancies the conductivity of $p$-sample was increased and their charges became negative and its energy levels shift up. In this case, positive vacancies transformed to negative by reconstructing broken bonds and getting energy for very fast diffusion [21]. Those excited negative vacancies, taking part in superdiffusion processes, were found in conductivity measurements of Si crystal irradiated by soft X-rays [6, 9].

Till temperatures about $800^{\circ} \mathrm{C}$ diffusion processes emanate through vacancies [22]. In this work we applied the superdiffusion of charged vacancies irradiated by soft X-rays for drive in of phosphorus and boron at room temperature, formerly introduced by thermal diffusion in crystal silicon. We obtained the important result that diffusion conditions and profiles in the irradiated samples is essentially different from the thermodiffusion conditions and profiles. The proposed new technologies open the possibility to drive in boron and phosphorus into silicon and can be used for production of microelectronics devices and solar cells.

\section{References}

[1] A.J. Janavičius, Acta Phys. Pol. A 93, 505 (1998).

[2] Seung-Man Park, Jae Mook Kim, Hee Lee, Choong-Ki Kim, Jpn. J. Appl. Phys. 35, L 1554 (1996).

[3] T. Wada, in: Proc. 3rd Int. Conf. Neutron Transmutation Doped Silicon, Copenhagen, 1981, Ed. T. Takagi, Institute of Electrical Engineering of Japan, Tokyo 1983, p. 447.

[4] T. Wada, H. Fujimoto, Phys. Status Solidi C 2, 788 (2003).

[5] R.D. Westbrook, T.L. Polgreen, in: IEEE Photovoltaic Specialists Conf., Baton Rouge 1976, (LA) USA, Conference Record. (A78-10902 01-44), Institute of Electrical and Electronics Engineers, Inc., New York 1976, p. 67.

[6] A.J. Janavičius, J. Banys, R. Purlys, S. Balakauskas, Lith. J. Phys. 42, 337 (2002).

[7] V.S. Vavilov, A.E. Kiv, O.R. Nijazova, Mechanism of Producing and Migration of Defects in Semiconductors, Science, Moscow 1981, p. 368. 
[8] A.J. Janavičius, R. Purlys, Ž. Norgèla, Acta Phys. Pol. A 109, 159 (2006).

[9] R. Purlys, A.J. Janavičius, A. Mekys, S. Balakauskas, J. Storasta, Lith. J. Phys. 41, 376 (2001).

[10] A.J. Janavičius, Ž. Norgèla, R. Purlys, Eur. Phys. J. Appl. Phys. 29, 127 (2005).

[11] A.J. Janavičius, Science and Technique 12, Journal of Lithuanian Academy of Science and Engineers Union, 19 (2005) (in Lithuanian).

[12] A. Strzałkowski, Introduction to Nuclear Physics, Panstwowe Wydawnictwo Naukowe, Warsaw 1978, p. 636 (in Polish).

[13] J.A. Van Vechten, Phys. Rev. B 10, 1482 (1974).

[14] A. Dargys, J. Kundrotas, Handbook on Physical Properties of Ge, Si, GaAs and InP, Science and Encyclopedia Publishers, Vilnius 1994, p. 262.

[15] A.J. Janavičius, Acta Phys. Pol. A 93, 731 (1998).

[16] A.J. Janavičius, Phys. Lett. A 224, 159 (1997).

[17] H.F. Matare, Defect Electronics in Semiconductors, Wiley-Interscience, New York 1971, p. 463.

[18] P.K. Kashkariov, Soros's Education Journal 1, 105 (1999).

[19] G.D. Watkins, Mater. Sci. Semicond. Proc. 3, 227 (2000).

[20] A.J. Janavičius, J. Storasta, R. Purlys, A. Mekys, S. Balakauskas, Ž. Norgèla, Acta Phys. Pol. A 112, 55 (2007).

[21] A.F. Chochlov, Physics of Solid State, Vol. 1, High School, Moscow 2001, p. 365 (in Russian).

[22] A.N. Bubenikov, Modeling Integral Microtechnologies, Devices and Schemes, Vysshaya Shkola, Moscow 1989, p. 320 (in Russian). 